\title{
Emotionsfokussierte Depressionstherapie: Pro und Contra
}

\begin{abstract}
Was wirkt wie und warum? Es liegt in der Natur der Sache, dass erfolgreiche Psychotherapieforschung nicht stehen bleibt, sondern die Interventionen und zugrundeliegenden theoretischen Konzepte differenziert überprüft und untersucht. Im Bereich der Depressionsforschung gibt es nachweislich wirksame kognitiv-verhaltenstherapeutische Interventionen. Ist eine Erweiterung/ Umbenennung/Ergänzung erfolgreicher Behandlungskonzepte notwendig? Professor Martin Hautzinger, der deutschsprachige Protagonist der kognitiven Verhaltenstherapie bei depressiven Störungen und Professor Martin Grosse Holtforth, SNF-Förderprofessor und Nachwuchswissenschaftler, vertreten ihre Positionen und regen mit ihren Statements zum Nachdenken über unsere therapeutischen Positionen an.
\end{abstract}

Ulrike Ehlert, Zürich

\section{+ Pro}

Die nachhaltige Wirksamkeit kognitiv-verhaltenstherapeutischer Depressionstherapien kann durch gezielte Förderung emotionaler Verarbeitung mit Hilfe emotionsfokussierter Interventionen gesteigert werden

Die Wirksamkeit kognitiv-verhaltenstherapeutischer Depressionstherapie zählt zu den stabilsten Befunden der Psychotherapieforschung [Hollon und Ponniah, 2010]. Nach aktuellem Kenntnisstand sind allerdings die mittel- und langfristigen Behandlungseffekte auch der erfolgreichsten Depressionstherapien klar verbesserungswürdig (z.B. 54\% Rückfälligkeit nach erfolgreicher kognitiver Therapie; [Vittengl, et al., 2007]). Um dieses drängende Problem zu lösen, verfolgt die Psychotherapieforschung bisher vor allem zwei Strategien: Während einige Forscher versuchen, die Erfolge der Akuttherapie durch Erhaltungstherapien (z.B. achtsamkeitsbasierte Therapie) zu stabilisieren, setzen andere an einer verbesserten Nachhaltigkeit der Akuttherapie an.

Wir nehmen an, dass die kognitiv-verhaltenstherapeutische Depressionstherapie bislang hinter ihren Möglichkeiten als Akuttherapie zurück bleibt, und ihre Nachhaltigkeit durch die gezielte Förderung emotionaler Verarbeitung mit
Hilfe emotionsfokussierter Interventionen optimieren kann. Leitidee für unsere Überlegungen ist das Duale-ProzessModell der Depression von Beevers [2005], wonach sowohl explizite (d.h. bewusst und kontrolliert ablaufende) als auch implizite (d.h. automatisch und assoziativ ablaufende) Prozesse an den depressiv verzerrten Verarbeitungsprozessen beteiligt sind. Der kognitive Ansatz [z.B. Wilken, 2006] sieht die Möglichkeit der Intervention vor allem auf der Ebene expliziter Kognitionen, die hier als Triebfeder für unerwünschte emotionale Zustände angenommen werden. Durch kognitive Interventionen wird zwar nachgewiesenermaßen erfolgreich eine Symptomreduktion erreicht, ob jedoch pathologische Verzerrungen der Informationsverarbeitung auf impliziter/automatischer Ebene verändert werden, bleibt zumindest fraglich [Glashouwer und De Jong, 2010; Risch et al., 2010]. Beevers [2005] hat deswegen eine Depressionstherapie vorgeschlagen, welche zusätzlich zu der Veränderung expliziter Prozesse durch kognitive Interventionen auch auf die Veränderung impliziter und assoziativer Informationsverarbeitungsprozesse abzielt. Im Sinne einer interozeptiven Exposition sollen sich die Patienten wiederholt absichtsvoll den Inhalten ihres depressiven Erlebens aussetzen und dabei unter therapeutischer Anleitung neue (v.a. emotionale) Erfahrungen machen, schrittweise weniger verzerrte Assoziationen zwischen Situationen und kognitivemotionalen Reaktionen auf Situationen lernen, das Neuge-

\section{KARGER}

Fax +497614520714

Information@Karger.de

www.karger.com

\section{(C) 2011 S. Karger GmbH, Freiburg}


lernte daraufhin explizit/reflektiv verarbeiten und mit der Zeit in neue emotional-kognitiven Schemata integrieren [Samoilov und Goldfried, 2000].

Über welche Interventionen erreicht man also eine Veränderung impliziter assoziativer Verarbeitungsprozesse und wie könnten kognitive Verhaltenstherapeuten diese in ihre Arbeit integrieren? Hier bieten sich vor allem Interventionen aus der von Hollon und Ponniah [2010] als «möglicherweise wirksam» klassifizierten emotionsfokussierten Therapie [Greenberg und Watson, 2005] an. Das Prinzip der Emotionstransformation beschreibt darin einen Prozess der Veränderung implizitassoziativer Verbindungen zwischen Situationen und Emotionen/Kognitionen. Prinzip der Emotionstransformation ist es, Emotionen mit Emotionen zu verändern. So soll eine stabile dysfunktionale emotionale Reaktion dadurch verändert werden, dass sie in eine funktionalere emotionale Reaktion umgewandelt wird. Beispielsweise soll ein lähmendes Gefühl der Wertlosigkeit gezielt - z.B. mit Hilfe einer Zweistuhltechnik - in der Therapiesitzung evoziert werden, damit der Patient sich der beteiligten psychologischen Prozesse (z.B. harsche Selbstkritik) bewusst wird. Ungleich der kognitiven Therapie, die an dieser Stelle den Wahrheitsgehalt dieser kritischen Selbstverbalisation in Frage stellen würde, soll eine emotionale Repräsentation des zugrundeliegenden Bedürfnisses (z.B. wertgeschätzt, geliebt zu werden) herausgearbeitet werden. Die Aktivierung dieses Bedürfnisses soll dann die Möglichkeit eröffnen, eine adaptive Emotion (z.B. Trauer um die nie erhaltene Wertschätzung durch die Eltern) bewusst zu erleben, die einen produktiven Umgang mit ursprünglich depressogenen Situationen ermöglichen soll. Wie das Beispiel zeigt, geht es bei der Emotionstransformation nicht darum, positiv zu denken, oder durch Denken negative Emotionen zu reduzieren, sondern darum, schrittweise adaptive Gefühle zu erleben und sie immer besser als Hinweisgeber für zentrale Bedürfnisse lesen zu lernen.

Einige kognitiv-verhaltenstherapeutische Behandlungsansätze integrieren bestimmte emotionszentrierte Interventionen bereits. So hat etwa die Exposition in sensu in der Trauma- und Angsttherapie eine lange, erfolgreiche Geschichte, und die Auffassung, dass Emotionen auch in kognitivverhaltenstherapeutisch orientierten Behandlungen aufgegriffen, akzeptiert, genutzt und bearbeitet werden sollen, gehört zunehmend zum therapeutischen «common sense». Entsprechend sieht das Unified Protocol zur Behandlung von Angstund affektiven Störungen nach Barlow [Barlow, et al., 2011] emotionszentrierte Interventionen als festen Bestandteil vor. Jedoch mangelt es hier und in den meisten Behandlungsmanualen an konkreten Anleitungen, den aufgeführten Veränderungsmechanismus der Emotionstransformation mit gezielten Interventionen umzusetzen.

Ein Beispiel für die Bearbeitung sowohl expliziter als auch impliziter Verzerrungen der Informationsverarbeitung in der Depressionstherapie ist die expositionsbasierte kognitive Therapie für Depressionen [EBCT-R; Grosse Holtforth, et al., zur
Veröffentlichung eingereicht; weiterentwickelt nach Hayes et al., 2007]. Während in der ersten Phase dieser manualisierten Depressionstherapie Stabilisierung und Stärkung des Patienten (z.B. durch Aktivitätsaufbau und Achtsamkeitsübungen) im Vordergrund stehen, sollen in der zweiten Phase darauf aufbauend depressogene emotionale Schemata transformiert werden (über die Konfrontation mit dem eigenen negativem Selbstbild und emotionsfokussierte Techniken), um in der letzten Phase eine Festigung der neuen, adaptiven Schemata zu fördern und die Rückfallprophylaxe aktiv zu planen.

\section{Martin Grosse Holtforth, Zürich}

\section{Literatur}

Barlow DH, Farchione TJ, Fairholme CP, Ellard KK, Boisseau CL, Allen LB, Ehrenreich-May J: Unified Protocol for Transdiagnostic Treatment of Emotional Disorders: Therapist Guide. New York, NY, Oxford University Press, 2011.

Beevers CG: Cognitive vulnerability to depression: A dual process model. Clin Psychol Rev 2005;25:975-1002.

Glashouwer KA, De Jong PJ: Disorder-specific automatic self-associations in

depression and anxiety: results of The Netherlands Study of Depression and Anxiety. Psychol Med 2010;40:1101-1111

Greenberg L, Watson J: Emotion Focused Therapy of Depression. Washington, DC, APA, 2005.

Hayes A, Feldman GC, Beevers, CG, Laurenceau, JP, Cardaciotto LA, Lewis-Smith

J: Discontinuities and cognitive changes in an exposure-based cognitive therapy for depression. J Consult Clin Psychol 2007;75:409-421.

Hollon SD, Ponniah K: A review of empirically supported psychological therapies for mood disorders in adults. Depress Anxiety 2010;27:891-932.

Risch, AK, Buba, A, Birk, U, Morina N, Steffens M, Stangier, U: Implicit self-esteem

- in recurrently depressed patients. J Behav Ther Exp Psychiatry 2010;41:199-206.

Samoilov A, Goldfried MR: Role of emotion in cognitive behavior therapy. Clin

Psychol: Science Practice 2000;7:373-385.

Vittengl JR, Clark LA, Dunn TW, Jarrett RB: Reducing relapse and recurrence in unipolar depression. J Consult Clin Psychol 2007;75:475-488.

Wilken, B: Methoden der kognitiven Umstrukturierung. Ein Leitfaden für die psychotherapeutische Praxis. ed 3. Stuttgart, Kohlhammer, 2006.

\section{Contra}

\section{Vorbemerkung}

Die Psychotherapie, damit auch die kognitive Verhaltenstherapie bei (akuten, chronischen, rezidivierenden) Depressionen zeigen zwar erfreuliche Effekte (Besserung, Remission, Erhaltung, Rezidivprophylaxe) und gute Effektstärken ( $\mathrm{d}=$ 0,9-1,6), doch sind die Erfolgsraten (45-60\% remittierter bzw. rückfallfreier Patienten) noch keineswegs befriedigend [Hollon et al., 2005; Cuijpers et al. 2008]. Die erste und naheliegende Lösung für dieses Problem ist die Entwicklung neuer und «innovativer» Interventionen. Es wird angenommen, dass sich die Erfolgsraten durch neue, alternative bzw. verbesserte Therapiestrategien verbessern lassen. Doch es darf bezweifelt werden, dass dies durch neue Therapietechniken und Behandlungsansätze zu erreichen ist. Was vielmehr fehlt ist 
die konsequente Umsetzung und Anwendung bewährter Behandlungsstrategien in der Praxis (Optimierung) sowie Forschung zur differenziellen Indikation (Moderatoren), um darüber zu verbesserten Behandlungsentscheidungen (Indikation) zu gelangen. Es ist eher der einfache Weg, neue Interventionen zu ersinnen, diese als «dritte Welle» zu vermarkten und $\mathrm{zu}$ behaupten, dass damit die Erfolgsrate gesteigert werden kann. Uns fehlen nicht kreative Vorschläge, sondern überzeugende Evidenzen für die Berechtigung dieser Neuentwicklungen.

\section{These 1: Die Wirksamkeit einer Psychotherapie bei Depressionen wird durch handlungs- und verhaltensbezogene Interventionen gesteigert. Dies gilt insbesondere bei schweren und chronifizierten Depressionen}

Insbesondere durch die Impulse und Vorarbeiten von Peter Lewinsohn sowie des späten Neil Jacobson liegen heute Studien vor [Jacobson et al., 1996; Dimidjian et al., 2006; Dobson et al., 2008], die zeigen, dass die konsequente Anwendung verhaltensaktivierender und -ändernder Interventionen («behavioural activation»), im Vergleich mit kognitiven Interventionen bzw. mit komplexeren Therapieprogrammen oder Antidepressiva zu deutlich besseren Ergebnissen mit längerfristiger Stabilität (bis zu 2 Jahren [Dobson et al., 2008]) führen (Abb. 1). Patienten mit schweren Depressionen, in der Regel mit Antriebsstörungen und deutlicher Anhedonie profitieren besonders von diesen strukturierenden, den Alltag gestaltenden, neues Verhalten einübenden Verfahren. Diese Erkenntnis deckt sich mit den klinischen Erfahrungen im stationären Rahmen. Auch dort weiß man schon lange, dass die Reflexion über Gefühle bzw. die Analyse von Einstellungen, automatischen Gedanken und Schemata in der Behandlung dieser schwer depressiven Patienten wenig zielführend ist. Vielmehr

Abb. 1. Prozentuale Anteile remittierter Patienten, gemessen mit der HamiltonDepressions-RatingSkala (HDRS) und dem Beck-Depressions-Inventar (BDI) in den Bedingungen Pharmakotherapie (SSRI), Kognitive Therapie (KT) und Verhaltensaktivierung (VT). Der Unterschied zwischen VT und SSRI bzw. KT ist signifikant $(\mathrm{p}<0,05)$ [nach Dimidjian et al. 2006]. $\square$ HDRS \% Remission $\quad$ BDI \% Remission

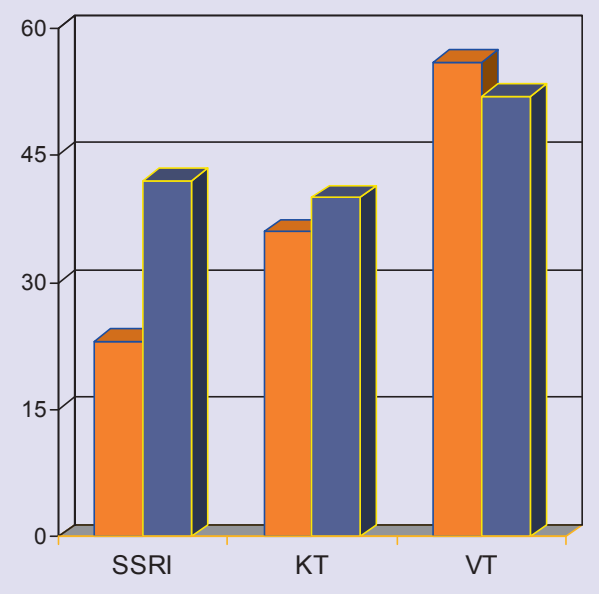

sind die klare, entlastende Alltagsstruktur, die festen Abläufe und die Einbindung in angenehme Tätigkeiten, Aktivitäten und Handlungen auch ohne innere Beteiligung entscheidend.

Im ambulanten Rahmen ist zu beklagen, dass diese verhaltensbezogenen Maßnahmen oft gar nicht bzw. unzureichend lang oder nur halbherzig angewandt werden. Es wird viel zu schnell und dann fast ausschließlich über Gedanken, Einstellungen, Erfahrungen, Gefühle und Befindlichkeiten gesprochen. Selbst CBASP (Cognitive Behavioral Analysis System of Psychotherapy)-Therapeuten, so meine Beobachtung, sind viel eher geneigt, die Therapiezeit mit Übertragungshypothesen, früh prägenden Bezugspersonen («Stempel») und disziplinierter Selbsteinbringung zu verbringen, als mit verhaltensnahen, im konkreten Alltag ansetzenden, handlungsbezogenen Situationsanalysen, obgleich diese 3/4 der therapeutischen Arbeit mit den chronifizierten Patienten ausmachen sollen. Folglich sollte eine gute, prognostisch erfolgreiche Depressionstherapie weniger emotionsfokussiert und kognitiv, als vielmehr verhaltensaktivierend und übend sein.

\section{These 2: Die Behauptung nachhaltigerer Wirksamkeit von kognitiver Verhaltenstherapie durch Förderung emotionsfokussierter Interventionen ist spekulativ. Ebenso die Behauptung, dass Emotionsfokussierung der entscheidende Wirkmechanismus der Psycho- therapie bei Depressionen sei}

Bis heute liegen keine kontrollierten Interventionsstudien zur emotionsfokussierten Psychotherapie bei Depressionen vor. - Geschweige denn Studien, die in einem direkten Vergleich emotionsfokussierte Psychotherapie mit Verhaltenstherapie oder anderen bewährten Psychotherapien verglichen. Wir wissen, dass depressive Patienten in hohem Maß durch sogenannte unspezifische Therapiefaktoren (Beziehungsvariablen, Erwartungen, Entlastungen, Erklärungen, Unterstützung usw.) erreichbar sind. Nicht nur Medikamentenstudien gelingt es schwer, Effektunterschiede zwischen Verum und Placebo aufzuzeigen, auch Psychotherapiestudien gelingt es oft nicht, für spezifische Behandlungen einen Wirkvorteil gegenüber unterstützenden Maßnahmen zu belegen. Es ist daher anzunehmen, dass die emotionsfokussierte Psychotherapie zwar gegenüber keiner Behandlung bzw. Wartelistenkontrollen erfolgreich abschneidet, sie jedoch kaum höhere Effektstärken erzielt als die bereits bestehenden, bewährten und evidenzbasierten Psychotherapien, wie VT, KVT, IPT. Das ist natürlich genauso eine Spekulation, wie z.B. die Behauptung, Psychoanalyse sei langfristig erfolgreicher als Verhaltenstherapie.

Es ist weiterhin höchst spekulativ, also noch nicht experimentell und mittels prospektiven Designs geprüft, in der Emotionsfokussierung einen entscheidenden Mediator des Therapieerfolgs zu sehen. Es ist jedoch den Vertretern dieses Ansatzes daraus kein Vorwurf zu machen, da die Wirkmechanismen und Mediatoren erfolgreicher (kognitiver) Verhal- 
tenstherapie bei Depressionen ebenfalls kaum bekannt sind. Die Erforschung von Wirkmechanismen setzt jedoch zunächst die evidenzbasierte Etablierung einer erfolgreichen Psychotherapie voraus, bevor die Verbindung von Grundlagenforschung und klinischer Interventionsforschung möglich wird.

\section{Schlussbemerkung}

Die Diskussion um verhaltensaktivierende, kognitive, emotionsfokussierte usw. Therapien erinnert mich stark an die Diskussion in den 70er und 80er Jahren des letzten Jahrhunderts über das Primat von Kognition bzw. Emotion. Über Monate, ja Jahre hinweg stritten etwa Lazarus und Zaijonc (z.B. im American Psychologist) darüber, was zuerst kommt, die Emotionen oder die Kognitionen. Aus der (europäischen) Distanz verstand man bald nicht mehr, worüber die beiden sich eigentlich stritten, denn es wurde immer offensichtlicher, dass beide völlig aneinander vorbei redeten. Die beiden Streithähne hatten vergessen, sich auf ein gemeinsames Verständnis der benutzten Begriffe zu verständigen. Diese Gefahr besteht bei der Pro-und-Contra-Debatte über alte und neue Psychotherapien, über kognitive und emotionsfokussierte Interventionen auch. Meist werden die dabei benutzen Begriffe unscharf verwendet bzw. die Auflösungsebene der Argumentation ist unterschiedlich, woraus sich möglicherweise scheinbare Gegensätze ergeben.

Martin Hautzinger, Tübingen

\section{Literatur}

Cuijpers P, van Straten A, Andersson G, van Oppen P: Psychotherapy for depres-

sion in adults: a meta-analysis of comparative outcome studies. J Consult Clin Psychol 2008;76:909-922.

Dimidjian S, Hollon S, Dobson K, Schmaling K, Kohlenberg R, Addis M et al.: Ran-

domized trial of behavioural activation, cognitive therapy, and antidepressant medication in the acute treatment of adults with major depression. J Consult Clin Psychol 2006;74:658-670.

Dobson K, Hollon S, Dimidjian S, Schmaling K, Kohlenberg R, Gallop R et al.:

Randomized trial of behavioural activation, cognitive therapy, and antidepressant medication in the prevention of relapse and recurrence in major depression. J Consult Clin Psychol 2008;76:468-477.

Hollon S, Jarrett R, Nierenberg A, Thase M, Trivedi M, Rush A: Psychotherapy and

medication in the treatment of adult and geriatric depression. J Clin Psychiatry 2005;66:455-468.

Jacobson N, Dobson K, Truax P, Addis M, Koerner K, Gollan J et al.: A component

analysis of cognitive behavioral treatment for depression. J Consult Clin Psychol 1996;64:295-304. 